\title{
Concepciones de la Enseñanza y el Aprendizaje en Profesorado de Educación Superior
}

\section{Conceptions of Teaching and Learning in Higher Education Teachers}

\author{
Fuensanta Hernández Pina, Javier J. Maquilón Sánchez, Mari Paz García Sanz \\ y Fuensanta Monroy Hernández \\ Universidad de Murcia-España
}

\begin{abstract}
Resumen. Las concepciones sobre la enseñanza y el aprendizaje que los profesores sostienen en su tarea docente es una línea de investigación que está suponiendo un avance en el conocimiento sobre factores relevantes para la mejor de la calidad en educación superior. Desde hace más de tres décadas han sido numerosos los investigadores que han venido proporcionando resultados de investigación que han llevado a crear un corpus de conocimientos sólidos sobre lo que hacen y piensan los profesores sobre su tarea docente. En el trabajo que presentamos se aborda lo que un grupo de docentes piensan sobre la enseñanza y el aprendizaje. El análisis, aunque de carácter cuantitativo, se ha basado en un cuestionario elaborado ad hoc en base a las propuestas realizadas por autores que han realizado sus estudios desde una metodología cualitativa. Los resultados apuntan hacia una concepción más cuantitativa que cualitativa, tanto en lo que se refiere a la enseñanza como al aprendizaje. Palabras clave: Educación Superior, concepciones de la enseñanza, concepciones del aprendizaje.
\end{abstract}

\begin{abstract}
Conceptions of teaching and learning held by teachers is a research topic which could mean a step forward in our understanding of important factors for improving the quality of Higher Education. For over three decades, a number of researchers have achieved results through studies which offer a corpus of solid knowledge about what teachers do and think about their teaching process. In this paper, we present the ideas held by a group of teachers about teaching and learning. The analysis, although quantitative in nature, is based on an ad hoc questionnaire based on the proposals made by some authors who have carried out studies using qualitative methodology. Results point towards a conception of the process with a quantitative profile rather than qualitative, with regard to both teaching and learning. Key words: Higher Education, conceptions of teaching, conceptions of learning.
\end{abstract}

\section{Introducción}

El estudio de las concepciones de la enseñanza y del aprendizaje en educación superior está siendo un tema de interés especial en el contexto de los nuevos planteamientos del proceso de Bolonia. La revisión

La correspondencia sobre este artículo dirigiarla a la primera autora. Dep. de Métodos de Investigación y Diagnóstico en Educación. Facultad de Educación. Universidad de Murcia. E-mail: fhpina@um.es de la literatura sobre este tema señala que tanto estudiantes como profesorado sostienen formas distintas en cuanto a la forma de percibir cómo debe ser la enseñanza y el aprendizaje en el escenario del aula

Este trabajo es el resultado de la investigación "Diseño, aplicación y evaluación de un programa de intervención para la mejora de la calidad del aprendizaje en estudiantes universitarios de nuevo ingreso". Financiada por la Comunidad Autónoma de la Región de Murcia, Proyectos Séneca, 2003. PB/38/FS/02. 
pese a que sus experiencias hayan podido ser similares o idénticas. Biggs (1999), uno de los autores que más ha contribuido a esta línea de investigación, expresa que los estudiantes y el profesorado pueden compartir experiencias de enseñanza y de aprendizaje similares pero mantener concepciones distintas ante ambas situaciones. Trigwell y Prosser (1991), por su parte, señalan que incluso puede darse una relación entre las creencias que el profesorado puede sostener sobre la enseñanza y el aprendizaje y sus prácticas dentro del aula. Del mismo modo autores como Marton y Saljo (1984), Van Rossum y Schenk (1984), Ramsden (1989), defienden que también los estudiantes podrían organizar las tareas de aprendizaje en función de sus concepciones personales de la enseñanza y el aprendizaje.

Los últimos 20 años han sido muy fructíferos en cuanto a la investigación en educación superior sobre esta temática dando lugar a un corpus de conocimientos y resultados que han permitido desarrollar modelos que tratan de explicar cómo tiene lugar la enseñanza y el aprendizaje desde la perspectiva de los protagonistas, profesores y estudiantes (Hernández Pina, 2002, 2004; Hernández Pina et al (2005). Muchos de estos resultados de investigación podrían ayudar a dar las claves sobre las respuestas a los nuevos planteamientos que el EEES plantea en lo referente a nuevas formas de aprender y nuevas formas de enseñar. Ramsden (1987) es partidario de que las investigaciones sobre esta temática se realicen desde el contexto en el que tienen lugar los procesos de enseñanza-aprendizaje con el fin de que los resultados derivados de dichas investigaciones tengan una mejor aplicación y puedan usarse por el profesorado en su aula y de este modo promover de un modo más realista todos aquellos cambios que desea se produzcan en sus alumnos.

Las investigaciones realizadas sobre concepciones se han llevado a cabo bajo dos perspectivas. Estudios que examinan la enseñanza y el aprendizaje desde la perspectiva del investigador (Schommer, 1990) y estudios que describen las experiencias del aprendizaje y la enseñanza desde la perspectiva de los protagonistas, como son los estudios desde la fenomenográfica de Marton, Dall'Alba y Beaty (1993). En esta segunda corriente los investigadores han analizado la variedad de significados que el aprendizaje y la enseñanza tienen para sus protagonistas y los modos diferentes de cómo aprenden y enseñan, utilizando para ello métodos cualitativos. Dentro de esta línea cualitativa de investigación queremos destacar los trabajos de Dall'Alba (1990); Fox (1983); Dunkin (1990); Trigwell et al. (1994); Martin and Bella (1990); Dunkin and Precian (1992); Gow and Kember (1994); Martin and Ramsden (1992); Prosser et al. (1994); Samuelowicz and Bain (1992). Algunas de estas investigaciones han llegado a la conclusión de que el profesorado puede enfocar su enseñanza centrándose en la materia o en sí mismo con el objetivo de transmitir información a los estudiantes, o bien, enfocarla sobre el estudiante con el objetivo de cambiar o desarrollar la comprensión que el estudiante tiene del mundo que le rodea (Prosser et al, 2005; Hernández Pina y Maquilón Sánchez, 2010).

Las creencias parecen jugar un papel fundamental en la toma de decisiones que los profesores realizan sobre la relevancia que unos conocimientos determinados pueden tener en un momento determinado (Pajares, 1992). Los datos aportados por investigadores como el citado apuntan a que hay una asociación entre las creencias epistemológicas y las concepciones de la enseñanza. Un profesor que sostiene una epistemología simplista podría creer que el conocimiento es simplista, claro y específico y la habilidad para aprenderlo por parte de sus alumnos sería innata y establecida. Por el contrario, un profesor que sostiene una creencia sofisticada del conocimiento se inclinará más por creer que el conocimiento es complejo, incierto y tentativo y, por tanto, poder construirse de forma gradual por parte del alumno. Hofer y Pintrich (1997) y Prawat (1992) con sus trabajos han contribuido a mostrar cómo las concepciones del profesor pueden llegar a afectar sus prácticas dentro del aula.

La revisión de algunos de los trabajos publicados sobre esta temática apuntan a unas taxonomías que trata de poner orden sobre las concepciones a las que 
los profesores pueden adscribirse (Gow y Kember, 1993; Prosser, Samuelowicz y Bain, Trigwell et al 1994; Murray y Macdonald, 1997; Martin, Prosser y Trigwell, Ramsden y Benjamin, 2000; Dall’Alba, 1990, Dunkin, 1990; Dunkin y Precian, 1992; Fox, 1983, 1983; Gow y Kember, 1993; Martin y Ramsden, 1992; Prosser et all, 1994). Estas taxonomías han sido resumidas en categorías organizadas jerárquicamente. Independientemente del número de categorías, su orientación se centra en el profesor o en el estudiante. La mayoría de estos trabajos han sido llevados a cabo en países con un entorno socioeconómico similar y en materias más relacionadas con el ámbito de las ciencias que las ciencias sociales y humanas.

En cuanto al número de categorías propuesto, los autores abogan por dos categorías, como es la propuesta de Gow y Kember (1993), o las siete de Samuelowicz y Bain (1992). En la mayor parte de los casos, aunque se hace uso de terminologías diferentes, la semántica es similar ya que las categorías indican o bien la importancia de la transmisión del conocimiento e información, o bien la construcción y desarrollo del estudiante. En términos genéricos, las categorías se centran en el profesor o en el alumno.

En lo que se refiere a la relación entre las categorías todos vienen a coincidir en que dicha relación es jerárquica bien de forma exclusiva y progresiva como proponen Dall'Alba (1991) y Martin y Balla (1991) o inclusiva como propone Samuelowicz. En esta relación inclusiva las categorías más altas incluyen los aspectos de las categorías más bajas.

Tal y como ya hemos descrito en otro lugar (Hernández Pina y Maquilón Sánchez, 2010), las categorías con una orientación centrada en la enseñanza y en el profesor tendrían como característica más destacada que la enseñanza se percibe como la transmisión de conocimientos. Los profesores en esta orientación se centrarían fundamentalmente en los contenidos de las materias y en lo que están enseñando, cómo organizarían, estructuraría y presentarían los contenidos de las materias para facilitar el aprendizaje. En cambio, las categorías con una orientación centrada en el aprendizaje y en el estudiante enfocarían la enseñanza como el apoyo al estudiante para que realice cambios conceptuales. Los profesores estarían más por la labor de lograr que sus alumnos construyeran su propio conocimiento y su comprensión y la promoción de su propia concepción (Samuelowicz y Bain, 1992, 2001, Prosser et al., 1994, Trigwell y Prosser, 1996b).

Uno de los artículos que consideramos seminales en esta línea de investigación es el artículo publicado por Kember en 1997. Este autor hace un análisis de la mayor parte de los trabajos (13 en total) llevados a cabo hasta esa fecha sobre concepciones de la enseñanza. En la síntesis de sus análisis Kember propone cinco categorías:

Dos centradas en el profesor como son la impartición de la información y transmisión de los conocimientos de forma estructurada.

Una denominada intermedia como es la interacción profesor-estudiante (apprenticeship).

Y otras dos centradas en el estudiante como son la facilitación de la comprensión y cambio conceptual y desarrollo intelectual.

En síntesis, las aportaciones parecen indicar que hay dos claras orientaciones con subcategorías variables. La primera es una orientación centrada en el profesor con un enfoque en la comunicación de los conocimientos y los contenidos de las materias. La segunda orientación estaría centrada en el estudiante y, por tanto, en el aprendizaje; centrada en el conocimiento.

El segundo concepto del título de nuestro estudio hace referencia a las concepciones del aprendizaje. Sobre esta línea de trabajo la literatura es mucho más amplia y el tiempo dedicado a su investigación data de los años 70. En el estudio sobre las concepciones del aprendizaje encontramos dos líneas de investigación claramente diferenciadas. Por un lado, la perspectiva epistemológica y, por otro, la perspectiva fenomenológica.

La perspectiva epistemológica se inicia en los años 70 gracias a los numerosos estudios llevados a cabo en el campo educativo. En esta línea de investigación la metodología utilizada ha sido la denomina- 
da de primer orden, es decir, la realizada desde la perspectiva de los investigadores (Marton et al., 1997). La corriente cognitivista describe el aprendizaje como un proceso de adquisición. La corriente constructivista da un paso más y concibe el conocimiento como algo que se construye y no como algo que se descubre. Esta perspectiva, al estudiar el aprendizaje, ha aportado resultados muy importantes que han permitido el desarrollo de teorías que hacen más comprensivo cómo se produce el aprendizaje. Hofer y Pintrich (1997) señalan que existe una relación entre las creencias epistemológicas acerca del conocimiento y las concepciones de aprendizaje.

Los resultados de las investigaciones parecen indicar que los profesores también despliegan niveles de desarrollo diferentes en su forma de percibir el aprendizaje. En educación superior los profesores en su forma diferente de actuar influyen en el desarrollo epistemológico que los estudiantes pueden ir manifestando en distintas situaciones de aprendizaje Schommer (1990), Perry (1981).

Uno de los trabajos pioneros en las investigaciones sobre aprendizaje han sido los llevados a cabo por Marton, Dall' Alba y Beaty (1993) y Ramsden (1992). Al hablar de las concepciones señalan la orientación reproductora y la orientación al cambio. La primera se basa en la memoria y la segunda en la construcción y el significado. Svensson (1984) lo ve en términos de cómo se organiza la información: discreta y segmentada o como un todo, atomística u holística. Dweck y Leggett (1988) señalaban también que las creencias epistemológicas influyen en la actuación académica. En síntesis, los estudios indican que hay una estrecha relación entre las creencias epistemológicas sobre el conocimiento y las concepciones que se desarrollan en el aprendizaje. Säljö (1979) fue uno de los pioneros en definir las concepciones diferenciando 5 categorías a las que Marton y cols. (1993) añadieron una sexta.

1. El aprendizaje es adquisición cuantitativa de conocimientos.

2. El aprendizaje es memorización.

3. El aprendizaje es adquisición de hechos y procedimientos para utilizar cuando se necesitan.

4. El aprendizaje sirve para comprender o extraer significados.

5. El aprendizaje es un proceso de interpretación y captación de la realidad.

6. El aprendizaje sirve para cambiar a la persona.

Las tres primeras tienen un carácter cuantitativo y el resto cualitativo (Watkins, 2001). La modulación de estas concepciones va a depender fundamentalmente del contexto donde tenga lugar el aprendizaje.

Nuestro trabajo parte de las propuestas de los autores citados haciendo una síntesis de las categorías propuestas maximizando su simplificación en cuanto a la definición de las categorías y de su número. Para ello hemos elaborado un cuestionario con 10 preguntas que trata de recoger las grandes categorías comentadas en referencia a las dos concepciones: por un lado, las dos concepciones centradas en el profesor y la materia y, por otra, las dos centradas en el aprendizaje y el estudiante; 4 preguntas se refieren a la enseñanza y 4 al aprendizaje. Se ha incluido una quinta y décima preguntas que sintetizan la viabilidad de una de las cuatro opciones en el contexto educativo presente.

El propósito del estudio ha sido analizar las concepciones que los profesores tienen sobre la enseñanza y el aprendizaje en el contexto de la institución en la que enseñan. Para ello hemos planteado como objetivos específicos analizar las concepciones de la enseñanza y del aprendizaje del profesorado en la muestra general de estudio y en función de la titulación en la que imparten docencia.

\section{Método}

\section{Participantes}

En la investigación han participado un total de 35 profesores de las titulaciones de Derecho (10), Biología (13) y Pedagogía (12). 


\section{Instrumentos}

Aunque la entrevista ha sido el recurso más utilizado para la recogida de datos en ambientes naturales de aula, en base a los trabajos revisados y al igual que hiciera Trigwell y Prosser $(1994,1996)$ hemos utilizado un cuestionario para analizar las concepciones de los profesores en base a las categorías propuestas en los estudios previamente analizados. Con el fin de establecer la validez y fiabilidad del cuestionario utilizado hemos procedido a realizar un ACP del cuestionario elaborado para este trabajo.

El cuestionario aplicado se denomina La Calidad del Aprendizaje de los Estudiantes. Cuestionario aplicado al profesorado CAEU-P. Este cuestionario consta de 22 ítems, de los cuales 5 hacen referencia a las concepciones de la enseñanza y otras 5 al aprendizaje. Para el presente trabajo sólo analizamos estas últimas 10 preguntas referidas a la enseñanza y al aprendizaje desde la perspectiva del profesorado. Cada pregunta se acompañó de una escala Likert de 5 valores en función del grado de acuerdo o desacuerdo que los participantes podían expresar con la afirmación presentada.

El cuestionario tenía el siguiente esquema:

\section{Enseñar es:}

1. Transmitir información acerca de los conceptos de la materia.

2. Ayudar a los estudiantes a adquirir conocimientos mediante una presentación organizada de los contenidos de la materia.

3. Facilitar el aprendizaje de los estudiantes mediante el desarrollo de estrategias para la comprensión de la materia.

4. Ayudar a los estudiantes a conseguir un cambio en su comprensión del mundo a través del cambio intelectual y la construcción de nuevos conocimientos.

5. Teniendo en cuenta el contexto en el que se desarrolla su enseñanza, de las cuatro opciones presentadas anteriormente ¿Cuál le parece la más viable?

\section{Aprender es:}

6. Adquirir información y herramientas conceptuales acerca de los contenidos de la disciplina.

7. Asimilar los conceptos de la disciplina y entender cómo estos se estructuran y relacionan.

8. Desarrollar competencias básicas para la comprensión y aplicación de la información, construyendo e interpretando los significados de los conocimientos adquiridos.

9. Transformar, cambiar las formas de ver el mundo, ser personas conscientes de su aprendizaje, creadoras de su realidad, fomentado la metacognición y promoviendo el desarrollo personal.

10. A partir del contexto en el que sus estudiantes aprender, de las cuatro opciones presentadas ¿Cuál cree es más factible de llevar a cabo?

Las preguntas 1, 2, 6 y 7 hacen referencia a concepciones cuantitativas. Las preguntas 3, 4, 8 y 9 a concepciones cualitativas. Las preguntas 5 y 10 se refieren a la viabilidad de una u otra en el contexto educativo presente.

\begin{tabular}{lccc}
\hline & CUANTITATIVA & CUALITATIVA & GLOBAL \\
\hline ENSEÑANZA & 1,2 & 3,4 & 5 \\
APRENDIZAJE & 6,7 & 8,9 & 10 \\
\hline
\end{tabular}

Con el fin de facilitar la presentación sólo se indicará los números de las preguntas.

Como sucede en numerosos estudios de pruebas educativas el cálculo del coeficiente de fiabilidad se basan en una única aplicación y obteniendo los coeficientes de fiabilidad de Kuder-Richardson y Cronbach. En nuestro caso hemos calculado la fiabilidad de la prueba total y separando los ítems referidos a las dos concepciones resultando una fiabilidad media para la prueba total y bastante aceptable para las subescalas por separado tal y como queda reflejado en la tabla 1.

Otra de las cuestiones planteadas ha sido el análisis de la estructura factorial de los ítems en su totalidad y las subescalas que lo componen. Para ello hemos efectuado dos tipos de análisis de componen- 
Tabla 1: Coeficientes de fiabilidad. *Excepto ítems 5 y 10

\begin{tabular}{ccccccc}
\hline & & Enseñanza & & \multicolumn{2}{c}{ Enseñanza } \\
\hline Total & Escala & Subescala & Subescala & Escala & Subescala & Subescala \\
\hline $1-10^{*}$ & $1-4$ & $1-2$ & $3-4$ & $6-9$ & $6-7$ & $8-9$ \\
.410 & -.009 & .731 & .537 & -.057 & .819 & .688 \\
\hline
\end{tabular}

Tabla 2: Cuestionario total (ítems 1-4 y 6-9)

\begin{tabular}{lcc}
\hline Preguntas & I & II \\
\hline 1. enseñanza por transmisión & .793 & .164 \\
2. enseñanza por facilitación & .711 & .497 \\
3. enseñanza por comprensión- & .508 & .592 \\
4. enseñanza por cambio & -.660 & .363 \\
6. aprendizaje por transmisión & .851 & .301 \\
7. aprendizaje por facilitación & .695 & .554 \\
8. aprendizaje por comprensión & -.581 & .684 \\
9. aprendizaje por cambio & -.761 & .246 \\
\hline TOTAL 70.46\% & $49.41 \%$ & $21.05 \%$ \\
\hline
\end{tabular}

tes principales: uno de primer orden referido a los ítems y otro de segundo orden referido a las subescalas y a las escalas. En la tabla 2 podemos apreciar los resultados de dicho análisis y la estructura del cuestionario.

Ambos factores expresan la bipolaridad de las concepciones con valores altos y positivos en las preguntas referidas a las concepciones cuantitativas y los otros valores también altos pero con valores negativos.

El siguiente análisis efectuado ha sido el relacionado exclusivamente con los ítems sobre la enseñanza. El resultado muestra claramente la bipolaridad de las concepciones (Tabla 3 ).

Similares resultado hemos obtenido al analizar las preguntas referidas al aprendizaje (Tabla 4).

A modo de conclusión, señalamos que el cuestionario ha resultado ser suficientemente fiable y con

Tabla 3: Cuestiones enseñanza (1 -4)

\begin{tabular}{lc}
\hline Preguntas & Componente \\
\cline { 2 - 2 } & 1 \\
\hline Enseñanza por transmisión & .830 \\
Enseñanza por facilitación & .708 \\
Enseñanza por comprensión & -.636 \\
Enseñanza por cambio & -.649
\end{tabular}

Tabla 4: Cuestiones aprendizaje profesores 6-9

\begin{tabular}{lcc}
\hline Preguntas & \multicolumn{2}{c}{ Componente } \\
\cline { 2 - 3 } & 1 & 2 \\
\hline 6 Aprendizaje por transmisión & .889 & .250 \\
7 Aprendizaje por facilitación & .703 & .637 \\
8 Aprendizaje por comprensión & -.574 & .734 \\
9 Aprendizaje por cambio & -.815 & .306 \\
\hline
\end{tabular}

una estructura clara sobre las concepciones cualitativa y cuantitativa, lo que ha permitido analizar los objetivos que nos hemos propuesto en el presente estudio.

\section{Procedimiento}

El procedimiento seguido en el trabajo de campo se inició con las oportunas gestiones con los decanos y el profesorado para el posterior envío de los cuestionarios. Una vez cumplimentados por los profesores se recogieron personalmente con el fin de obtener información complementaria sobre algunos de los aspectos abordados en dicho cuestionario. Las titulaciones donde se aplicaron dichos cuestionarios fueron Derecho, Pedagogía y Biología.

Una vez recogida la información, se procedió a analizar los datos con el paquete estadístico SPSS, versión 12.0 .

\section{Resultados y discusión}

El objetivo inicial planteado ha sido analizar las concepciones de la enseñanza y del aprendizaje del profesorado de educación superior de las tres titulaciones mencionadas. 
En primer lugar analizamos las concepciones manifestadas por el profesorado a nivel general. Sobre la concepción 1 , el $37.1 \%$ del profesorado está de acuerdo en que la enseñanza debe ser la transmisión de información. Si a este porcentaje le unimos las respuestas de la escala 4 y 5 observamos que el $68.6 \%$ opinan que esto debe ser así y sólo el $31.5 \%$ ha manifestado no estar de acuerdo.

En la concepción 2 observamos que el 57\% está de acuerdo y totalmente de acuerdo en que la enseñanza debe traducirse en ayudar a los estudiantes a adquirir conocimientos. Sólo el $14.3 \%$ dice no estar de acuerdo.

Las respuestas dadas a la concepción 3 indican claramente la opinión del profesorado $(42.9 \%)$ de que enseñar debe servir para facilitar el aprendizaje de los estudiantes. Sólo el 17.2\% manifiesta no estar de acuerdo.

En la concepción 4 llama la atención la ausencia de respuestas a las opciones uno y dos. Todo el profesorado ha manifestado estar en acuerdo o totalmente de acuerdo en que enseñar debe servir para prestar ayuda a los estudiantes a conseguir un cambio en la comprensión del mundo que les rodea y a construir nuevos conocimientos.

Cuando le hemos preguntado al profesorado sobre cuál de la cuatro concepciones es la más viable en el contexto presente, el $54.3 \%$ opina que debe ser (concepción 2) la de ayudar a los estudiantes a adquirir conocimientos mediante la presentación organizada de los contenidos de la materia. Si a esta opción unimos la uno, el porcentaje sube a $80 \%$, lo que indica una posición claramente cuantitativa de la enseñanza como es la transmisión y la adquisición de conocimientos.

Igualmente le hemos preguntado al profesorado qué es para ellos aprender. Se les han presentado cuatro opciones. Dos de carácter cuantitativo y dos de carácter cualitativo. Las respuestas han sido las siguientes:

El 68.6\% del profesorado está de acuerdo y bastante de acuerdo en que aprender es adquirir información y herramientas conceptuales acerca del contenido de la disciplina. Solo el $31.5 \%$ dice no estar de acuerdo.

Cuando la opción de la concepción (7) ha sido la asimilación de conceptos, el $88.6 \%$ dice estar de acuerdo o totalmente de acuerdo con que la enseñanza debe cumplir dicho fin. Sólo el $11.4 \%$ del profesorado dice no estar de acuerdo que el aprender tenga que producirse así.

Cuando se le ha planteado si aprender puede desarrollar competencias para la comprensión, el 97\% está de acuerdo o en total acuerdo con dicha concepción. Apenas un 3\% dice no estar de acuerdo.

Lo mismo sucede cuando la concepción (9) hace referencia a que aprender sea transformar, cambiar, promover el desarrollo personal. El $96.3 \%$ está de acuerdo en que eso debe ser aprender.

Ahora bien, cuando a la vista del contexto y la realidad institucional se le ha preguntado cuál de las concepciones es la más viable para sus estudiantes a la hora de llevar a cabo el aprendizaje, el $71.4 \%$ está de acuerdo en que debería hacerse a través de las concepciones 6 y 7 . Es decir, el $34.3 \%$ adquirir información y el $37.1 \%$ asimilar conceptos.

Tabla 5: Profesores Total

\begin{tabular}{lcccc}
\hline & $\begin{array}{c}\text { 1. Enseñanza por } \\
\text { transmisión }\end{array}$ & $\begin{array}{c}\text { 2. Enseñanza por } \\
\text { facilitación }\end{array}$ & $\begin{array}{c}\text { 3. Enseñanza por } \\
\text { comprensión }\end{array}$ & $\begin{array}{c}\text { 4. Enseñanza por } \\
\text { cambio }\end{array}$ \\
\hline 6. Aprendizaje por transmisión & $.756^{* *}$ & $.622^{* *}$ & -.142 & $-.446^{* *}$ \\
& .000 & .000 & .417 & .007 \\
7. Aprendizaje por facilitación & $.492^{* *}$ & $.755^{* *}$ & -.155 & $.373^{* *}$ \\
8. Aprendizaje por comprensión & .003 & .000 & $.670^{*}$ & .091 \\
9. Aprendizaje por cambio & $-.344^{*}$ & -.138 & .000 & $.470^{* *}$ \\
& $.043^{* *}$ & .431 & .323 & .004 \\
& $-.462^{* *}$ & $-.376^{*}$ & .058 & $.049^{* *}$ \\
\hline
\end{tabular}




\section{Coherencia}

Hemos tratado de ver la coherencia entre las respuestas dadas por el profesorado en las preguntas 14 y 6-9 a partir de la correlación entre estas opciones. Los resultados son reveladores. Los coeficientes de correlación han sido presentados en la tabla 5.

Los coeficientes obtenidos indican una coherencia interna entre ítems pertenecientes a la misma escala, significativa al nivel de confianza del $.01 \mathrm{y}$ .05. Los coeficientes dentro de la misma escala han resultado coeficientes positivos y los de escalas diferentes, negativos.

Es destacable la coherencia entre las concepciones 1 y 6 donde se decía que la enseñanza debe ser transmisión de información y el aprendizaje adquisición de información. Y las opciones 4 y 9 que rezan que la enseñanza debe ayudar a lo estudiantes a conseguir un cambio en su comprensión del mundo y el aprendizaje debe servir para transformar, cambiar. Los que opinan cuantitativamente o cualitativamente lo hacen en ambas concepciones a la vez y de forma coherente.

Los coeficientes de correlación entre las opciones dentro de la enseñanza y dentro del aprendizaje muestran la intensidad de la coherencia en la forma de opinar de los profesores. Los coeficientes entre las opciones cuantitativas son significativos y altos y las opciones cuantitativas con las cualitativas son significativos y altos también, pero con signo negativo.

$\mathrm{Al}$ correlacionar las preguntas 5 y 10 observamos un coeficiente de .688 , significativo al .01. Este coeficiente indica una alta coherencia entre las concepciones sobre la enseñanza y el aprendizaje que los profesores consideran más viables de acuerdo con el contexto actual. En este caso parece que lo viable son las concepciones cuantitativas tanto de la enseñanza como del aprendizaje.

El segundo objetivo ha sido analizar las concepciones de la enseñanza y del aprendizaje del profesorado en función de la titulación en la que imparten docencia. Los resultados han sido como sigue:

\section{Profesorado de Biología}

En la concepción uno, el 53.8\% está de acuerdo en que enseñar es transmitir información. El 53.8\% también opina que debe ayudar a adquirir los conocimientos de la materia. El 53.8\% cree igualmente que debe facilitar el aprendizaje y, por último, el $46.2 \%$ dice que debe ayudar a conseguir un cambio en su comprensión del mundo. Cuando la pregunta ha sido qué opción de las cuatro considera más viable de acuerdo con el contexto institucional, la respuesta ha sido clara y contundente, el $76.9 \%$ opta por la opción dos y el $23.1 \%$ por la uno. Este resultado indica una posición claramente por una enseñanza de corte cuantitativo.

En las respuestas sobre qué entienden debe ser el aprendizaje las respuestas a las cuatro opciones han sido para la concepción uno el $46.2 \%$ está de acuerdo en que debe servir para adquirir información. En cambio, el $61.5 \%$ está bastante de acuerdo en que sea asimilación de conceptos de la disciplina (concepción 2). En la concepción 3, el $46.2 \%$ está totalmente de acuerdo en que debe servir para el desarrollo de competencias y en la concepción 4, el 38.5\% opina que debe servir para la transformación y el cambio. Cuando la pregunta ha sido qué opción de las cuatro considera más viable de acuerdo con el contexto institucional la respuesta ha sido clara y contundente, el $53.8 \%$ se orienta por la concepción dos y el $30.8 \%$ por la uno. Este resultado indica una clara posición por un aprendizaje cuantitativo. Globalmente diremos que la coherencia entre las concepciones de enseñanza y aprendizaje han arrojado un coeficiente de .424 , aunque no significativo.

\section{Profesorado de Derecho}

Los datos referidos al profesorado de derecho los analizamos con cierta cautela dado el numero (10) de participantes. Sin embargo, estos resultados pueden estar expresando una tendencia que no queremos dejar de analizar. En la concepción uno, el 80\% del profesorado está de acuerdo con que la enseñan- 
za debe ser transmisión de información. En la pregunta 2 , el $90 \%$ está totalmente de acuerdo que debe servir para ayudar a los estudiantes a adquirir conocimientos. En las preguntas cualitativas los resultados indican que todos los participantes están de acuerdo en mayor o menor grado que la enseñanza debe ser facilitación del aprendizaje (concepción 3) y ayudar a los estudiantes a conseguir cambios en su comprensión del mundo (concepción 4). Cuando la pregunta ha sido sobre qué concepción de las cuatro considera más viable de acuerdo con el contexto institucional, la respuesta ha sido clara y contundente, el $40 \%$ optan por la concepción uno y el $30 \%$ por la dos. Este resultado $(70 \%)$ indica una clara posición por una enseñanza con perfil cuantitativo.

En las respuestas sobre qué entienden que debe ser el aprendizaje las respuestas a las cuatro concepciones han sido las siguientes: para la concepción uno, el $60 \%$ está de acuerdo que debe servir para adquirir información. En cambio, el 50\% está bastante de acuerdo en que sea la asimilación de los conceptos de la disciplina. En la concepción 3, el $40 \%$ está de acuerdo en que debe ser el desarrollo de competencias y en la concepción 4, el $40 \%$ opina que debe ser la transformación y el cambio. Cuando la pregunta ha sido qué opción de las cuatro considera más viable de acuerdo con el contexto institucional, la respuesta ha sido clara y contundente. El $60 \%$ optan por la concepción uno y el $10 \%$ por la dos, lo que de forma conjunta acumula un $70 \%$ que optan por un planteamiento cuantitativo. Este resultado indica una clara tendencia por un aprendizaje cuantitativo. Globalmente diremos que la coherencia entre las concepciones de enseñanza y aprendizaje ha sido un coeficiente significativo de .743 .

\section{Profesorado de Pedagogía}

En el análisis de las respuestas dadas por el profesorado de Pedagogía observamos los resultados siguientes. En la concepción uno el $33.3 \%$ está en desacuerdo en que enseñar es transmitir información. El 33.3\% está bastante de acuerdo en que debe servir para ayudar a adquirir conocimientos. El 41.7\% está bastante de acuerdo en que debe servir para facilitar el aprendizaje y, por último, el $75 \%$ que debe ayudar a conseguir un cambio en su comprensión del mundo. Cuando la pregunta ha sido qué opción de las cuatro considera más viable de acuerdo con el contexto institucional, la respuesta ha sido clara y contundente. El $66.7 \%$ opta por la concepción dos y el $16.7 \%$ por la uno. Este resultado indica una clara tendencia por una enseñanza de corte cuantitativo.

En las respuestas sobre qué entienden que debe ser el aprendizaje las respuestas a las cuatro opciones han sido. Para la concepción 6, el 33.3\% está en desacuerdo que debe ser adquirir información. En cambio, el mismo porcentaje aparece en las categorías 2 desacuerdo y 4 bastante de acuerdo en que sea (concepción 7) la asimilación de los conceptos de la disciplina. En la concepción 8, el $58.3 \%$ está totalmente de acuerdo en que debe servir para el desarrollo de competencias y en la concepción 9, el $66.7 \%$ opina que debe ser la transformación y el cambio. Cuando la pregunta ha sido qué opción de las cuatro considera más viable de acuerdo con el contexto institucional, la respuesta ha sido clara y contundente. El 58.3\% optan por la concepción 7 y el $16.7 \%$ por la 6 . Este resultado indica una clara tendencia por un aprendizaje cuantitativo. Globalmente diremos que la coherencia entre las concepciones de enseñanza y aprendizaje ha sido un coeficiente significativo de .756 .

\section{Conclusiones}

La concepción de enseñanza más viable para los profesores es la concepción 2 que dice que debe servir para ayudar a los estudiantes a adquirir conocimientos mediante una presentación organizada de los contenidos de la disciplina. Para el aprendizaje la opción más viable considera que es la 7 que dice que el aprendizaje debe servir para asimilar los conceptos de la disciplina y entender/memorizar los contenidos y construirlos/relacionarlos con sus conocimientos e ideas previas. Esta preferencia viene ava- 
lada por el coeficiente de correlación entre las preguntas 5 y 10 que ha sido de $.688^{* *}$. A nivel de titulaciones las concepciones de enseñanza propuesta por los distintos grupos ha sido la 2 para los profesores de biología y pedagogía y la 1 para los de derecho. En cuanto a las concepciones de aprendizaje ha sido la 7 para los estudiantes de biología y pedagogía y la 6 para los de derecho. Los coeficientes de correlación entre las opciones más viables en su contexto han sido para biología .424, para pedagogía $.756^{* *}$ y para derecho $.742^{*}$, mostrando dichos coeficientes la coherencia de cada uno de las tres submuestras, altas, positivas y significativas para derecho y pedagogía y alta y no significativa para biología.

\section{Referencias}

Biggs, J. (1999). Teaching for quality learning at university. Buckingham: Society for Research in Higher Education and Open University Press.

Dall.Alba, G. (1991). Foreshadowing conceptions of learning. Research and Development in Higher Education, 13, 293-297.

Dunkin, M. J. (1990). The induction of academic staff to a university: Processes and products. Higher Education, 20, 47-66.

Dunkin, M. J. y Precians R.P. (1992). Award-winning university teachers' concepts of teaching. Higher Education, 24, 483-502.

Entwistle, N.J. (1987). A model of the teachinglearning process derived from research on student learning. En J. Richardson, M. Eysenck y D.W. Arren- Piper, (Eds.) Student learning research in education and cognitive psychology. London: University Press.

Fox, D. (1983). Personal theories of teaching. Studies in Higher Education, 8, 151-163.

Kember, D. (1997) A reconceptualization of the research into university academics conception of teaching. Learning and Instruction, 7, 255-275.

Gow, L. y Kember, D. (1990). Does higher education promote independent learning? Higher Education, 19, 307-322.
Gow, L., Kember, D. et al. (1992). Lecturers' views of their teaching practices: Implications for staff development needs. Higher Education Research and Development, 11, 135-149.

Gow, L. y Kember, D. (1993). Conceptions of teaching and their relationship to student learning. British Journal of Educational Psychology, 63, 20-33.

Hernández Pina, F. (2002a). La calidad del aprendizaje y la enseñanza en educación superior. Revista de Investigación Educativa, 19, 465-486.

Hernández Pina, F. (2002b). Docencia e investigación en educación superior. Revista de Investigación Educativa, 20, 271-301.

Hernández Pina, F. (2004). Enseñar y aprender en la Universidad: ¿Qué enseñar? ¿Qué aprender? Actas del III Symposium Iberoamericano de Docencia Universitaria Pedagogía Universitaria: Hacia un Espacio de Aprendizaje Compartido. ICE de Universidad de Deusto, pp.81-93.

Hernández Pina, F. y Maquilón Sánchez, J.J. (2010). Las concepciones de la enseñanza. Aportaciones para la formación del profesorado. REIFOP, 13 (3), 17-25. Enlace Web: http://www.aufop.com Hernández-Pina, F; Martínez Clares, P., Rosário, P. y Espín, M. (2005). Aprendizaje, competencias y rendimiento en Educación Superior. Madrid: La Muralla.

Hofer, B. K. y Pintrich, P. R. (1997). The development of epistemological theories: beliefs about knowledge and knowing and their relation to learning. Review of Educational Research, 67, $88-140$.

Kember, D. (1997). A reconceptualisation of the research into university academics. conceptions of teaching. Learning and Instruction, 7, 255275.

Kember, D. y Gow, L. (1994). Orientations to teaching and their effect on the quality of student learning. Journal of Higher Education, 65, 58-74.

Martin, E. y Balla, M. (1991). Conceptions of teaching and implications for learning. Research and Development in Higher Education 13, 298-304.

Martin, E. y Ramsden, P. (1992). An expanding 
awareness. How lecturers change their understanding of teaching. Higher Education, 13, 298-304.

Marton, F., Dall' Alba, G., \& Beaty, E. (1993). Conceptions of learning. International Journal of Educational Research, 19, 277-300.

Marton, F. y Saljo, R. (1984). Approaches to learning. En F. Marton, D. Hounsell and N.J. Entwistle (Eds). The Experience of Learning. Edinburgh: Scottish Academic Press.

Murray, K. y Macdonald, R. (1997). The disjunction between lecturers. conceptions of teaching and their claimed educational practice. Higher Education, 33, 331-349.

Pajares, M. F. (1992). Teachers. beliefs and education research: Cleaning up a messy construct. Review of Educational Research, 63, 307-332.

Perry, W. G. (1981). Cognitive and ethical growth: The making of meaning. En: A. W. Chickering (Ed.) The modern American college. San Francisco, CA: Jossey-Bass.

Prawat, R. S. (1992). Teachers beliefs about teaching and learning: a constructivist perspective. American Journal of Educational Psychologist, 100, 354-395.

Prosser, M.; Trigwell, K. et al. (1994). A phenomenographic study of academics' conceptions of science learning and teaching. Learning and Instruction, 4, 217-231.

Prosser, M.; Martin E. et al. (2005). Academics. experiences of understanding of their subject matter and the relationship of this to their experiences of teaching and learning. Instructional Science, $33,137-157$.
Ramsden, P. (1989). Perceptions of courses and approaches to studying: an encounter between paradigms. Studies in Higher Education, 14, 157-8.

Ramsden, P. (1992). Learning to teach in Higher Education. London and New York: Routledge.

Säljö, R. (1979). Learning about learning. Higher Education, 8, 443-451.

Samuelowicz, K. y Bain, J. D. (1992). Conception of teaching held by academia teachers. Higher Education, 24, 93-112.

Samuelowicz, K. y Brain, J. D. (2001). Revising academic beliefs about teachin and learning. Higher Education, 41, 299-325.

Schommer, M. (1990). The effect of beliefs about nature of knowledge on comprehension. Journal of Educational Psychology, 82, 498-504.

Trigwell, K. y Prosser, M. (1991). Improving the quality of student learning: the influence of learning context and student learning outcomes. Higher Education, 22, 251-266.

Trigwell, K.; Prosser, M. et al. (1994). Qualitative differences in approaches to teaching first year university science. Higher Education, 27, 85-93.

Trigwell, K y Prosser, M. (1996). Changing approaches to teaching: a relational perspective. Studies is Higher Education, 21, 275-284.

Van Rossum, E. J. y Schenk,S.M. (1984). The relationship between learning conception, study strategy and learning outcome. British Journal of Educational Psychology, 54, 73-83.

Watkins, D. (2001). Learning about learning enhances performance. National School Improvement Network Research Matters, 13, 15-37.

Manuscrito recibido: 10/09/2010

Revisión recibida: 18/10/2010

Manuscrito aceptado: 21/10/2010 